\title{
Utilization of echocardiography during septic shock was associated with a decreased 28-day mortality: a propensity score- matched analysis of the MIMIC-III database
}

\author{
Peng Lan ${ }^{1,2}$, Ting-Ting Wang ${ }^{2}$, Hang-Yang Li ${ }^{2}$, Ru-Shuang Yan ${ }^{2}$, Wei-Chao Liao ${ }^{2}$, Bu-Wen Yu ${ }^{2}$, \\ Qian-Qian Wang ${ }^{2}$, Ling Lin ${ }^{2}$, Kong-Han Pan ${ }^{2}$, Yun-Song Yu ${ }^{1}$, Jian-Cang Zhou ${ }^{2}$ \\ ${ }^{1}$ Department of Infectious Diseases, ${ }^{2}$ Department of Critical Care Medicine, Sir Run Run Shaw Hospital, College of Medicine, Zhejiang University, \\ Hangzhou 310016, China \\ Contributions: (I) Conception and design: P Lan and JC Zhou; (II) Administrative support: None; (III) Provision of study materials or patients: None; \\ (IV) Collection and assembly of data: HY Li, WC Liao; (V) Data analysis and interpretation: P Lan, TT Wang; (VI) Manuscript writing: All authors; \\ (VII) Final approval of manuscript: All authors. \\ Correspondence to: Jian-Cang Zhou. Department of Critical Care Medicine, Sir Run Run Shaw Hospital, No. 3 Qing Chun Road East, Hangzhou \\ 310016, China. Email: jiancangzhou@zju.edu.cn.
}

Background: Hemodynamic management is of paramount importance in patients with septic shock. Echocardiography has been increasingly used in assessing volume status and cardiac function. However, whether the utilization of echocardiography has an impact on prognosis is unknown. Thus, we intended to explore its effect on the outcomes of patients with septic shock.

Methods: The study was based on the Medical Information Mart for Intensive Care (MIMIC) III database. Septic shock patients were divided into two groups according to the usage of echocardiography during the onset of septic shock. The primary outcome was 28-day mortality. Secondary outcomes included the usage of inotropes, ventilation-free and norepinephrine-free time, and fluids input. Propensity-score matching was used to reduce the imbalance.

Results: Among 3,291 eligible patients, 1,289 patients who underwent echocardiography (Echo), and 1,289 who did not receive the Echo, had similar propensity scores and were included in the analyses. After matching, the Echo group had a significantly lower 28 -day mortality $(33.2 \%$ vs. 37.7\%, $\mathrm{P}=0.019)$. More patients in the Echo group received pulmonary artery catheter (PAC) $(4.2 \%$ vs. $0.2 \%, \mathrm{P}<0.001)$ and inotropes $(17.8 \%$ vs. $7.1 \%, \mathrm{P}<0.001)$. In the survival analysis, Echo utilization was associated with improved 28-day mortality [hazard ratio (HR): 0.83; 95\% confidence interval (CI), 0.73-0.95, P=0.005]. A reduced likelihood of 28-day mortality in patients with Echo $v s$. those without Echo was maintained either when excluding patients receiving multiple echocardiography scans (HR, 0.82; 95\% CI, 0.72-0.94; $\mathrm{P}=0.004$ ) or when excluding patients undergoing PAC or pulse index continuous cardiac output (PiCCO) (HR, 0.87; 95\% CI, 0.76-0.99; $\mathrm{P}=0.034)$.

Conclusions: Utilization of echocardiography was associated with improved 28-day outcomes in patients with septic shock.

Keywords: Echocardiography; propensity score matching; septic shock; mortality

Submitted Jun 22, 2019. Accepted for publication Sep 22, 2019.

doi: $10.21037 / \mathrm{atm} .2019 .10 .79$

View this article at: http://dx.doi.org/10.21037/atm.2019.10.79 


\section{Introduction}

Septic shock is one of the leading causes of death in critically ill patients, with mortality rates of approximately $40-50 \%$ (1-3). It is generally acknowledged that hemodynamic management is of paramount importance in patients with septic shock so as to guarantee organ perfusion and facilitate further titrate therapies (4). Although there are some ways to assess volume status and cardiac function in septic shock, few have been confirmed to actually improve patients' outcomes. Moreover, the commonly clinically used central venous pressure (CVP) has been shown to be poorly correlated with volume status or fluid responsiveness (5). Pulse index continuous cardiac output (PiCCO) and pulmonary artery catheter (PAC) are two invasive monitors that evaluate both preload and myocardial contractibility and help to guide therapy adjustment. However, PiCCO-based fluid management does not improve outcomes when compared to CVP-based fluid management (6). Likewise, PAC has been used less and less, as there has been no benefit from its use (7), and it has even been associated with an increase in mortality (8).

Echocardiography, a minimally invasive and repeatable hemodynamic monitoring tool, has become increasingly essential in the management of septic shock because it can not only help differentiate the causes of shock, but it can also provide real-time information of volume status and cardiac function $(3,7,9)$. It is the best bedside method to repeatedly assess cardiac function, and relevant guidelines have now recommended it as the first-line evaluation modality $(3,10)$. In a recent study, use of transthoracic echocardiography was found to be associated with an improved outcome in sepsis patients (11). Therefore, given the advantages of echocardiography in the management of shock, the likelihood that the outcomes would be improved for patients with septic shock when managed with echocardiography is an idea with the intuitive appeal, yet few empirical data is available regarding this. Thus, we hypothesized that echocardiography utilization during septic shock might decrease 28-day mortality for patients with septic shock because it may expedite hemodynamic stabilization.

\section{Methods}

\section{Data source}

This was a retrospective study based on a publicly available Medical Information Mart for Intensive Care (MIMIC)
III (version 1.4) ICU database $(12,13)$. It is a large, singlecenter database compromising the information of 46,520 critically ill patients admitted to the Beth Israel Deaconess Medical Center (Boston, Massachusetts) from 2001 to 2012 (13). Access to the database for research was approved by the Institutional Review Boards of the Massachusetts Institute of Technology (Cambridge, MA, USA) and the Beth Israel Deaconess Medical Center after completion of the NIH web-based course named "Protecting Human Research Participants". Given that all patients were deidentified, informed consent was waived by the ethical committee of the Beth Israel Deaconess Medical Center. Data were extracted by structured query language with pgAdmin4 PostgreSQL 9.6.

\section{Study population and definitions}

According to Angus criteria (14), all sepsis patients supported with norepinephrine within 24 hours after ICU admission were enrolled as septic shock patients in this retrospective study. For patients readmitted to the ICU, only the first ICU admissions were included. As echocardiography may also be used for the routine evaluation of ICU admissions rather than focusing on volume and cardiac performance assessment in patients with septic shock, we enrolled only those who underwent an echocardiography 24 hours within the onset of septic shock. Enrolled septic shock patients were divided into two groups according to the usage of echocardiography during the onset of septic shock; i.e., with echocardiography (Echo) and without Echo groups (Figure 1).

Patients' baseline characteristics, Sequential Organ Failure Assessment (SOFA) scores, Simplified Acute Physiology Score (SAPS) II, and Elixhauser comorbidity score was calculated as described in previous studies $(5,15,16)$. The primary endpoint was 28 -day mortality. The secondary endpoints included PAC and PiCCO administration, transfusion, usage of inotropes, norepinephrine-free, and ventilation-free days within 28 days after enrollment, and fluid input within 72 hours of septic shock occurrence.

\section{Statistical analysis}

Continuous variables were presented as medians with interquartile range (IQR), and categorical variables were presented by number and percentage. Continuous data were compared by the Mann-Whitney test, and categorical data were compared by chi-square or Fisher's exact test as 


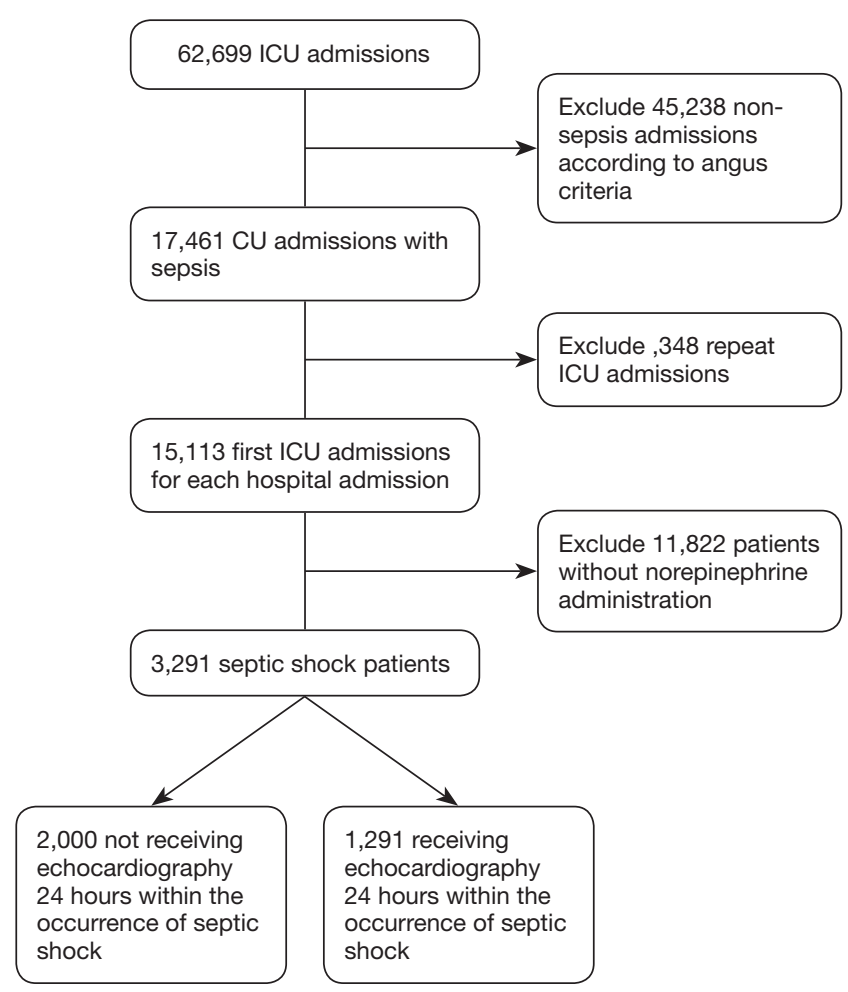

Figure 1 Flow chart: the inclusion of the study population.

appropriate. The distribution of the missing data was shown in Table S1.

To reduce the imbalance between the Echo and without Echo groups, propensity score analysis (PSA) was performed. Baseline characteristics including age, gender, weight, admission type (including elective, emergency, and urgent), positive blood culture, SOFA scores for each organ (i.e., respiration, renal, cardiovascular, liver, coagulation, and central nervous system), CVP, positive blood culture, metastatic cancer, complicated diabetes, hypertension, renal failure, congestive heart failure, mechanical ventilation, and ICU length of stay (LOS) were involved in the PSA. After matching, survival analysis was performed to determine whether the usage of echocardiography affected 28-day mortality. The effect of echocardiography was expressed by hazard ratio (HR) with $95 \%$ confidence interval (CI). Kaplan-Meier curves were depicted and compared by logrank test. Two sensitivity analyses were performed: (I) to determine if only one echocardiography scan, as opposed to its sequential use, can independently influence the outcomes by selecting patients who underwent a single echocardiography scan within 24 hours of septic shock occurrence, and (II) to exclude the confounding effect of
$\mathrm{PiCCO}$ and PAC by excluding those patients receiving either PiCCO or PAC.

All analyses were performed using R, version 3.3.3 for Windows (http://www.r-project.org/); a $\mathrm{P}$ value less than 0.05 was considered statistically significant.

\section{Results}

\section{Patient characteristics}

Patients who did not meet the Angus criteria, repeatedly admitted to ICU, or did not receive norepinephrine therapy were excluded. A total of 3,291 septic shock patients were included in the study, with 1,291 (39.2\%) receiving echocardiography within 24 hours of septic shock occurrence (Figure 1). Before propensity-score matching, gender, weight, admission type, SAPS II, SOFA score, congestive heart failure, metastatic cancer, CVP, positive rate of blood culture, and the proportion of mechanical ventilation usage were different between the two groups (Table 1). With the use of propensity-score matching (1:1 matching ratio), 1,289 patients who underwent echocardiography were matched with 1,289 patients who did not receive echocardiography. After matching, the imbalance between the Echo and without Echo groups was significantly reduced (Figure S1), and all the baseline variables were comparable between the two groups (Table 1).

\section{Outcome comparisons}

After matching, the Echo group had a significantly lower 28 -day mortality ( $33.2 \%$ vs. $37.7 \%, \mathrm{P}=0.019)$, and more patients in the Echo group received PAC (4.2\% vs. $0.2 \%$, $\mathrm{P}<0.001)$ and inotropes $(17.8 \%$ vs. $7.1 \%, \mathrm{P}<0.001$, Table 2$)$. However, there were no differences on the use of $\mathrm{PiCCO}$ and transfusion between the two groups. Those who received echocardiography had marginally longer norepinephrine durations while no difference was observed with mechanical ventilation durations. There was a trend that the patients in the Echo group received more fluid resuscitation, although it was not statistically significant (Table 2).

\section{Primary analysis}

To determine whether echocardiography was associated with the prognosis of septic shock patients, survival analysis was performed. As shown in Figure 2, echocardiography was 
Table 1 Baseline characteristics before and after propensity-score matching

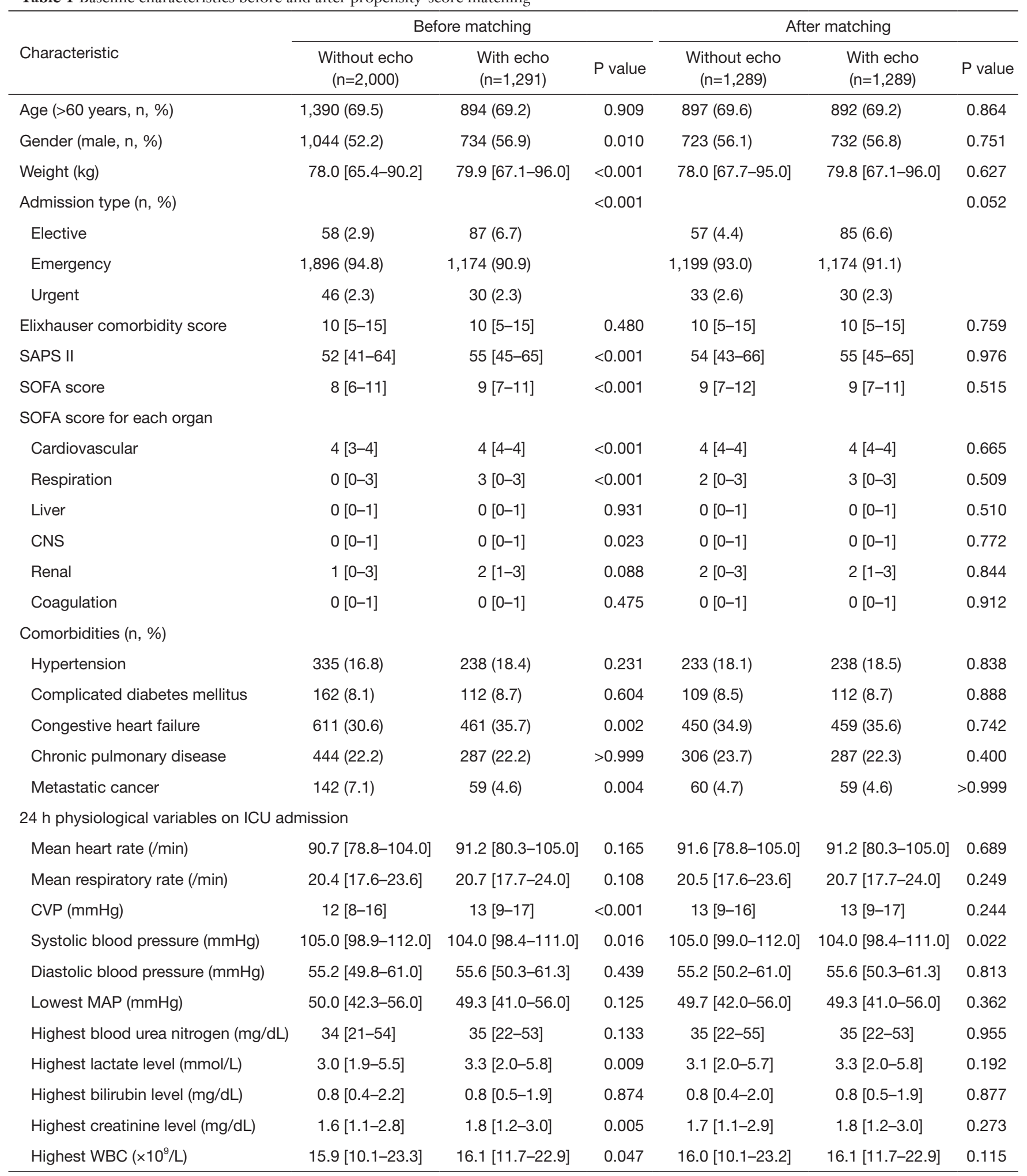

Table 1 (continued) 
Table 1 (continued)

\begin{tabular}{|c|c|c|c|c|c|c|}
\hline Characteristic & \multicolumn{3}{|c|}{ Before matching } & \multicolumn{3}{|c|}{ After matching } \\
\hline Urine output (mL) & $1,132[493-2,059]$ & $1,102[512-1,888]$ & 0.613 & $1,070[451-1,895]$ & $1,102[515-1,887]$ & 0.249 \\
\hline Positive blood culture (n, \%) & $422(21.1)$ & $223(17.3)$ & 0.008 & $221(17.1)$ & $223(17.3)$ & 0.958 \\
\hline Renal replacement therapy & $182(9.1)$ & $128(9.9)$ & 0.471 & $142(11.0)$ & $128(9.9)$ & 0.403 \\
\hline Mechanical ventilation & $1,284(64.2)$ & $1,004(77.8)$ & $<0.001$ & 997 (77.3) & $1,002(77.7)$ & 0.850 \\
\hline
\end{tabular}

Echo, echocardiography; SAPS, Simplified Acute Physiology Score; SOFA, Sequential Organ Failure Assessment; CNS, central nervous system; CVP, central venous pressure; MAP, mean arterial pressure; WBC, white blood cell.

Table 2 Outcomes of patients in matched cohort.

\begin{tabular}{|c|c|c|c|}
\hline Variable & Without echo $(n=1,289)$ & With echo $(n=1,289)$ & $P$ value \\
\hline 28-day mortality (n, \%) & $486(37.7)$ & $428(33.2)$ & 0.019 \\
\hline \multicolumn{4}{|l|}{ Secondary outcomes } \\
\hline $\operatorname{PAC}(n, \%)$ & $3(0.2)$ & $54(4.2)$ & $<0.001$ \\
\hline Transfusion (n, \%) & $299(23.2)$ & $294(22.8)$ & 0.852 \\
\hline Use of inotropes & $92(7.1)$ & $229(17.8)$ & $<0.001$ \\
\hline Norepinephrine-free days in 28 days & $26.9[25.5-27.6]$ & 26.7 [25.2-27.5] & 0.007 \\
\hline Ventilation-free days in 28 days & $21.0[5.3-25.9]$ & $20.8[6.9-25.6]$ & 0.446 \\
\hline Highest rate $(\mu \mathrm{g} / \mathrm{kg} / \mathrm{min})$ & $0.20[0.10-0.41]$ & 0.22 [0.10-0.40] & 0.167 \\
\hline \multicolumn{4}{|l|}{ Fluid input } \\
\hline $1^{\text {st }} 24 \mathrm{~h}$ after shock & $4,674[2,174-9,393]$ & $5,010[2,398-9,639]$ & 0.426 \\
\hline $2^{\text {nd }} 24 \mathrm{~h}$ after shock & $2,204[971-4,976]$ & $2,457[1,081-4,772]$ & 0.164 \\
\hline $3^{\text {rd }} 24 \mathrm{~h}$ after shock & $1,749[723-3,607]$ & $1,697[758-3,622]$ & 0.821 \\
\hline \multicolumn{4}{|l|}{ Urine output } \\
\hline $1^{\text {st }} 24 \mathrm{~h}$ after shock & $1,485[683-3,010]$ & 1,419 [619-2,792] & 0.359 \\
\hline $2^{\text {nd }} 24 \mathrm{~h}$ after shock & $1,802[833-3,162]$ & $1,570[756-2,887]$ & 0.091 \\
\hline
\end{tabular}

Echo, echocardiography; PAC, pulmonary artery catheter; PiCCO, pulse index continuous cardiac output. 
associated with improved 28-day mortality (HR: 0.83; 95\% CI, 0.73-0.95, $\mathrm{P}=0.005$ ).

To illuminate the effect of echocardiography on the outcomes of septic shock patients, two sensitivity analyses were performed. The first sensitivity analysis, which
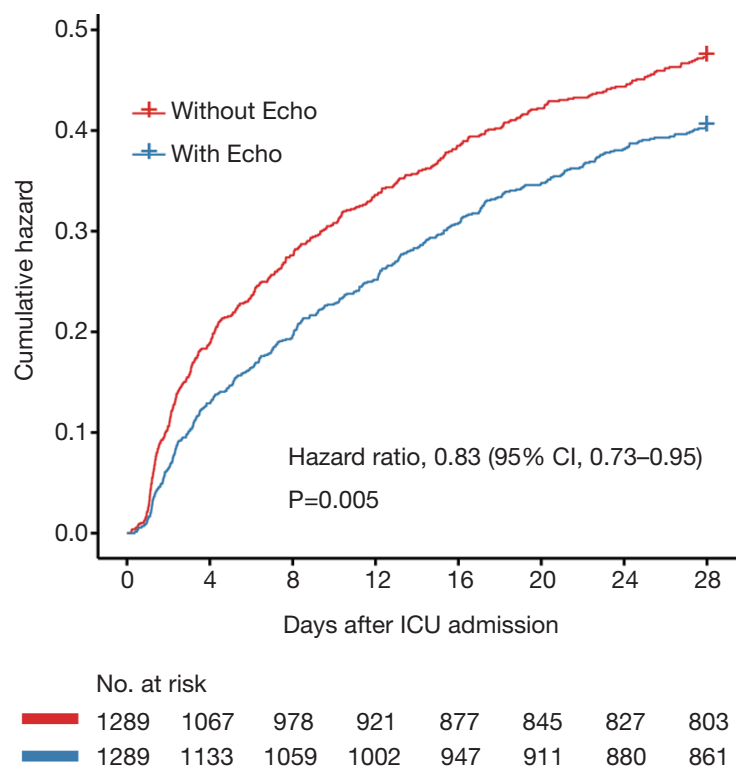

Figure 2 Cumulative hazard of 28-day mortality in the echo group $v s$. the without echo group.
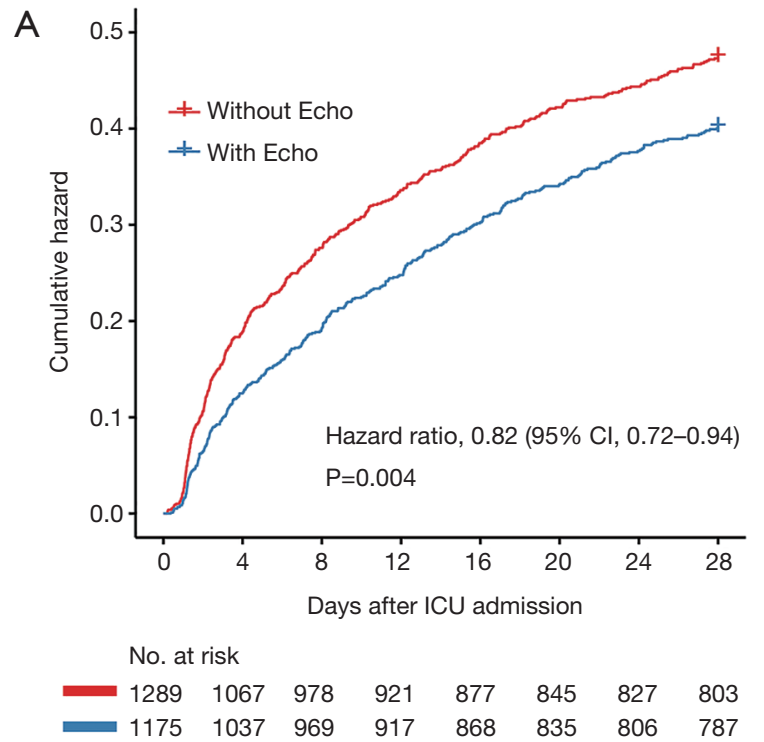

excluded 114 patients who underwent multiple sessions of echocardiography, identified 2,464 patients from the matched cohort. As shown in Figure $3 A$, a reduced likelihood of 28-day mortality in patients with Echo $v s$. those without Echo was maintained (HR, 0.82; $95 \%$ CI, 0.72-0.94; $\mathrm{P}=0.004)$. The second sensitivity analysis identified 2,485 patients who did not undergo PAC or PiCCO. The reduced likelihood of 28-day mortality in Echo group was maintained (HR, 0.87; 95\% CI, 0.76-0.99; $\mathrm{P}=0.034)$ (Figure 3B).

\section{Discussion}

Hemodynamic monitoring and assessment is a key component of the evaluation of critically ill patients, especially for those with septic shock, and bedside echocardiography has become increasingly crucial in the past years. To our knowledge, this is the first study evaluating the effect of echocardiography in the prognosis of patients with septic shock. The study demonstrated that utilization of echocardiography within 24 hours of septic shock occurrence was associated with a decreased 28-day mortality (HR: 0.83, $\mathrm{P}=0.005$ ).

In the USA, the reported prevalence of sepsis in ICUderived cohorts is $12 \%$ of all ICU admissions, with the hospital mortality for septic shock approaching 40-60\% (17).
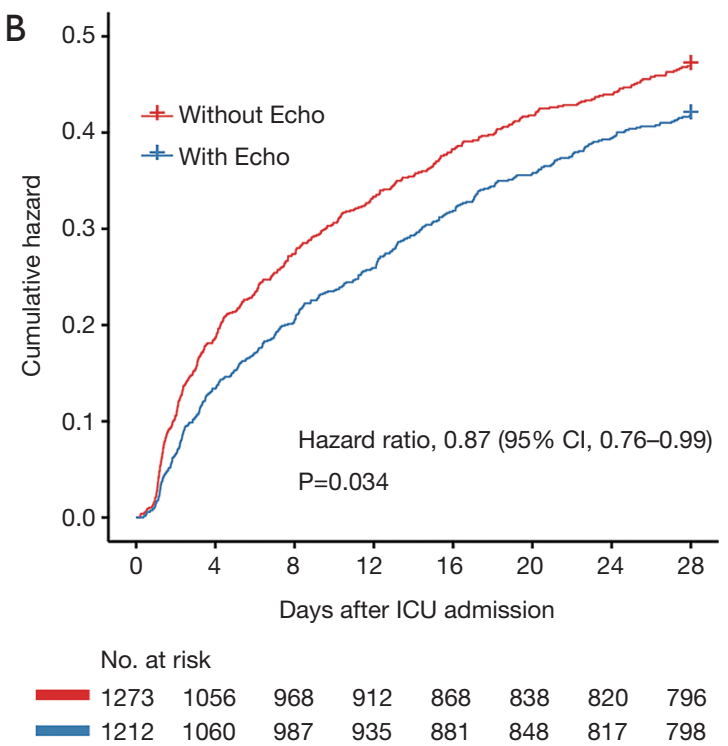

Figure 3 Cumulative hazard of 28-day mortality in the echo group $v$ s. the without echo group after (A) excluding patients who either received multiple sessions of echo or (B) or who received PiCCO or PAC. PiCCO, pulse index continuous cardiac output; PAC, pulmonary artery catheter. 
With strategies including early goal-directed therapy (EGDT), rapid empiric broad-spectrum antimicrobials therapy, and 3-h and 6-h bundles, the mortality of sepsis and septic shock has decreased steadily in recent years. Although the incidence of sepsis is increasing each year, the mortality of septic shock is still very high (18).

Early fluid resuscitation remained a cornerstone in the management of the septic shock for the restoration of tissue perfusion and the improvements of prognosis and was recommended as a critical therapy in the Surviving Sepsis Campaign (SSC) (4). Accurately assessing and monitoring the volume status and the response to a fluid challenge can effectively titrate fluid resuscitation. Monitoring strategies, including CVP, PAC, and PiCCO, are widely used for hemodynamic monitoring. However, the ability of CVP to predict a response to a fluid challenge when the CVP is within a relatively normal range $(8-12 \mathrm{mmHg})$ is limited (19). Therefore, the use of CVP alone to guide fluid resuscitation is not justified (3). PAC was previously shown to not significantly affect mortality and morbidity (20-22), and was even associated with more complications than CVC-guided therapy (23). Thus, PAC was strongly recommended against in routine use for patients with sepsis-induced ARDS in SSC 2016 (4). As for PiCCO, the requirement for intra-arterial and central venous catheterization limits the use to those with evolving critical illness or at high risk of complex and severe hemodynamic derangement (24). Furthermore, it has shown no benefit to patients' survival compared with CVP $(6,25)$. This is because simply using a tool like PAC or PiCCO will not improve outcomes; rather, the decisive issue is how clinicians use the obtained information to subsequently perform management.

Fortunately, echocardiography was recently demonstrated to be associated with an improvement in 28-day mortality in patients with sepsis (11). Theoretically, septic shock patients with compromised hemodynamic may benefit most from the guidance of Echo. As a widely utilized noninvasive monitoring tool, echocardiography was frequently reported to result in active changes in management (26), including fluid administration, inotrope or drug therapy, and treatment limitation (27). As reported, the utility of echocardiography was associated with the later initiation or increase of diuretics and inotropic support (27). A prospective study focusing on the effect of echocardiography on diastolic dysfunction in patients with septic shock demonstrated that echocardiography might identify those patients who require further fluid resuscitation during septic shock despite a CVP that indicates adequate resuscitation according to the current guidelines (28). In the 818 management actions resulted from echocardiography, fluid administration (32.4\%), vasopressor use (16.5\%), inotrope use $(11.7 \%)$ were the most frequently undertaken actions (29). Consistently, there was more inotrope and fluid input administered in the Echo group in our analysis. With this kind of active management driven by echocardiography, septic shock can be reversed (30). Additionally, due to the rapid and sequential evaluation of cardiac output, cardiac function, and preload, echocardiography has become the first-line tool to differentiate the cause of shock (31). Furthermore, myocardial strain or stress changes identified by echocardiography can reveal early sepsis-induced myocardial dysfunction. In summary, echocardiography can help the ICU physicians in three ways: (I) better characterization of the hemodynamic disorders; (II) selection of the best therapeutic options (intravenous fluids, inotropes, and ultrafiltration); (III) assessment of the response of the hemodynamic disorders to therapy (3).

However, the association between these subsequent changes in management and prognosis has yet to be conclusively demonstrated. In our study, patients with echocardiography were more clinically severe, which was consistent with a previous study (32). Septic shock patients who underwent Echo received more norepinephrine, fluid, and inotropes. More importantly, Echo was associated with a lower risk of death. Patients with sepsis may progress in disease severity from infection with a modest degree of organ dysfunction to septic shock (33). This chain of progression represents a window of opportunity, in which correct identification of the patients' condition and the appropriate interventions and monitoring are likely to improve outcomes (33). Therefore, we recommend the use of Echo for sepsis and septic shock patients in clinical practice.

Although the present study was the first to reveal the association between the use of echocardiography and the outcomes of septic shock patients, some limitations exist. Firstly, this was a retrospective study based on a singlecenter database, resulting in limited generalizability. Secondly, due to the limitation of the database, some clinical variables were missing, including detailed measurements from echocardiography reports. Thirdly, because of the nature of the retrospective analysis, the onset times of septic shock was not available. Thus, the time of initiation of norepinephrine served as the alternative. Lastly, our primary outcome was 28 -day mortality. There are significant 
outcomes variables that were not taken into consideration, including long-term mortality and ICU re-admission.

To conclude, in this large retrospective analysis, we demonstrated that echocardiography during septic shock resulted in subsequent active changes in management, including initiation of norepinephrine and inotropes. More importantly, echocardiography was associated with improved 28-day outcomes. Although this study is hypothesis-generating only, it does provide sufficiently strong evidence to justify a randomized controlled trial.

\section{Acknowledgments}

We would like to thank Prof. Yun-Xian Yu for statistical assistance.

Funding: This work was supported by Zhejiang Provincial Basic and Public Welfare Research Program (LGF19H150005) and Zhejiang Provincial Natural Science Foundation of China under Grant (LY16H150004).

\section{Footnote}

Conflicts of Interest: The authors have no conflicts of interest to declare.

Ethical Statement: The authors are accountable for all aspects of the work in ensuring that questions related to the accuracy or integrity of any part of the work are appropriately investigated and resolved. Access to the database for research was approved by the Institutional Review Boards of the Massachusetts Institute of Technology (Cambridge, MA, USA) and the Beth Israel Deaconess Medical Center.

\section{References}

1. Howell MD, Davis AM. Management of Sepsis and Septic Shock. JAMA 2017;317:847-8.

2. Liu V, Escobar GJ, Greene JD, et al. Hospital deaths in patients with sepsis from 2 independent cohorts. JAMA 2014;312:90-2.

3. Cecconi M, De Backer D, Antonelli M, et al. Consensus on circulatory shock and hemodynamic monitoring. Task force of the European Society of Intensive Care Medicine. Intensive Care Med 2014;40:1795-815.

4. Rhodes A, Evans LE, Alhazzani W, et al. Surviving Sepsis Campaign: International Guidelines for Management of Sepsis and Septic Shock: 2016. Intensive Care Med
2017;43:304-77.

5. Singer $M$, Deutschman CS, Seymour CW, et al. The Third International Consensus Definitions for Sepsis and Septic Shock (Sepsis-3). JAMA 2016;315:801-10.

6. Zhang Z, Ni H, Qian Z. Effectiveness of treatment based on PiCCO parameters in critically ill patients with septic shock and/or acute respiratory distress syndrome: a randomized controlled trial. Intensive Care Med 2015;41:444-51.

7. Johnson A, Mohajer-Esfahani M. Exploring hemodynamics: a review of current and emerging noninvasive monitoring techniques. Crit Care Nurs Clin North Am 2014;26:357-75.

8. Connors AF Jr, Speroff T, Dawson NV, et al. The effectiveness of right heart catheterization in the initial care of critically ill patients. SUPPORT Investigators. JAMA 1996;276:889-97.

9. Phillips RA, Hood SG, Jacobson BM, et al. Pulmonary Artery Catheter (PAC) Accuracy and Efficacy Compared with Flow Probe and Transcutaneous Doppler (USCOM): An Ovine Cardiac Output Validation. Crit Care Res Pract 2012;2012:621496.

10. Levitov A, Frankel HL, Blaivas M, et al. Guidelines for the Appropriate Use of Bedside General and Cardiac Ultrasonography in the Evaluation of Critically Ill Patients-Part II: Cardiac Ultrasonography. Crit Care Med 2016;44:1206-27.

11. Feng M, McSparron JI, Kien DT, et al. Transthoracic echocardiography and mortality in sepsis: analysis of the MIMIC-III database. Intensive Care Med 2018;44:884-92.

12. Goldberger AL, Amaral LA, Glass L, et al. PhysioBank, PhysioToolkit, and PhysioNet: components of a new research resource for complex physiologic signals. Circulation 2000;101:E215-20.

13. Johnson AE, Pollard TJ, Shen L, et al. MIMIC-III, a freely accessible critical care database. Sci Data 2016;3:160035.

14. Angus DC, Linde-Zwirble WT, Lidicker J, et al. Epidemiology of severe sepsis in the United States: analysis of incidence, outcome, and associated costs of care. Crit Care Med 2001;29:1303-10.

15. Le Gall JR, Lemeshow S, Saulnier F. A new Simplified Acute Physiology Score (SAPS II) based on a European/North American multicenter study. JAMA 1993;270:2957-63.

16. van Walraven C, Austin PC, Jennings A, et al. A modification of the Elixhauser comorbidity measures into a point system for hospital death using administrative data. Med Care 2009;47:626-33.

17. Cecconi M, Evans L, Levy M, et al. Sepsis and septic 
shock. The Lancet 2018;392:75-87.

18. Rhee C, Dantes R, Epstein L, et al. Incidence and Trends of Sepsis in US Hospitals Using Clinical vs Claims Data, 2009-2014. JAMA 2017;318:1241-9.

19. Eskesen TG, Wetterslev M, Perner A. Systematic review including re-analyses of 1148 individual data sets of central venous pressure as a predictor of fluid responsiveness. Intensive Care Med 2016;42:324-32.

20. Richard C, Warszawski J, Anguel N, et al. Early use of the pulmonary artery catheter and outcomes in patients with shock and acute respiratory distress syndrome: a randomized controlled trial. JAMA 2003;290:2713-20.

21. Rhodes A, Cusack RJ, Newman PJ, et al. A randomised, controlled trial of the pulmonary artery catheter in critically ill patients. Intensive Care Med 2002;28:256-64.

22. Shah MR, Hasselblad V, Stevenson LW, et al. Impact of the pulmonary artery catheter in critically ill patients: meta-analysis of randomized clinical trials. JAMA 2005;294:1664-70.

23. Wheeler AP, Bernard GR, Thompson BT, et al. Pulmonary-artery versus central venous catheter to guide treatment of acute lung injury. N Engl J Med 2006;354:2213-24.

24. Litton E, Morgan M. The PiCCO monitor: a review. Anaesth Intensive Care 2012;40:393-409.

25. Yuanbo Z, Jin W, Fei S, et al. ICU management based on PiCCO parameters reduces duration of mechanical ventilation and ICU length of stay in patients with severe thoracic trauma and acute respiratory distress syndrome. Ann Intensive Care 2016;6:113.

Cite this article as: Lan P, Wang TT, Li HY, Yan RS, Liao WC, Yu BW, Wang QQ, Lin L, Pan KH, Yu YS, Zhou JC. Utilization of echocardiography during septic shock was associated with a decreased 28-day mortality: a propensity score-matched analysis of the MIMIC-III database. Ann Transl Med 2019;7(22):662. doi: 10.21037/atm.2019.10.79
26. Mathru M. Transthoracic Echocardiography: Impact on Diagnosis and Management in Tertiary Care Intensive Care Units. Yearbook of Anesthesiology and Pain Management 2006;2006:154.

27. Orme RM, Oram MP, McKinstry CE. Impact of echocardiography on patient management in the intensive care unit: an audit of district general hospital practice. Br J Anaesth 2009;102:340-4.

28. Brown SM, Pittman JE, Hirshberg EL, et al. Diastolic dysfunction and mortality in early severe sepsis and septic shock: a prospective, observational echocardiography study. Crit Ultrasound J 2012;4:8.

29. Alherbish A, Priestap F, Arntfield R. The introduction of basic critical care echocardiography reduces the use of diagnostic echocardiography in the intensive care unit. J Crit Care 2015;30:1419.e7-1419.e11.

30. El-Nawawy AA, Abdelmohsen AM, Hassouna HM. Role of echocardiography in reducing shock reversal time in pediatric septic shock: a randomized controlled trial. J Pediatr (Rio J) 2018;94:31-9.

31. Vincent JL, De Backer D. Circulatory shock. N Engl J Med 2013;369:1726-34.

32. Sahin S, Uysal Yazici M, Ayar G, et al. Clinical impact and efficacy of bedside echocardiography on patient management in pediatric intensive care units (PICUs): A prospective study. Anatol J Cardiol 2017;18:136-41.

33. Perner A, Holst LB, Haase N, et al. Common Sense Approach to Managing Sepsis. Crit Care Clin 2018;34:127-38. 
Original Article

Table S1 Distribution of missing data (n, \%) for included variables

\begin{tabular}{|c|c|c|}
\hline Variables & Before matching $(n=3,291)(n, \%)$ & After matching $(n=2,578)(n, \%)$ \\
\hline Age & $0(0)$ & $0(0)$ \\
\hline Gender & $0(0)$ & $0(0)$ \\
\hline Weight & $159(4.8)$ & $112(4.3)$ \\
\hline Admission type & $0(0)$ & $0(0)$ \\
\hline \multicolumn{3}{|l|}{ Elective } \\
\hline \multicolumn{3}{|l|}{ Emergency } \\
\hline \multicolumn{3}{|l|}{ Urgent } \\
\hline Elixhauser comorbidity score & $0(0)$ & $0(0)$ \\
\hline SAPS II & $0(0)$ & $0(0)$ \\
\hline SOFA score & $0(0)$ & $0(0)$ \\
\hline \multicolumn{3}{|l|}{ SOFA score for each organ } \\
\hline Cardiovascular & $0(0)$ & $0(0)$ \\
\hline Respiration & $0(0)$ & $0(0)$ \\
\hline Liver & $0(0)$ & $0(0)$ \\
\hline CNS & $0(0)$ & $0(0)$ \\
\hline Renal & $0(0)$ & $0(0)$ \\
\hline Coagulation & $0(0)$ & $0(0)$ \\
\hline \multicolumn{3}{|l|}{ Comorbidities } \\
\hline Hypertension & $0(0)$ & $0(0)$ \\
\hline Complicated diabetes mellitus & $0(0)$ & $0(0)$ \\
\hline Congestive heart failure & $0(0)$ & $0(0)$ \\
\hline Chronic pulmonary disease & $0(0)$ & $0(0)$ \\
\hline Metastatic cancer & $0(0)$ & $0(0)$ \\
\hline \multicolumn{3}{|c|}{$24 \mathrm{~h}$ physiological variables on ICU admission } \\
\hline Mean heart rate & $1(0)$ & $1(0)$ \\
\hline Mean respiratory rate & $2(0)$ & $1(0)$ \\
\hline CVP & $1,210(36.8)$ & $854(33.1)$ \\
\hline Lowest MAP & $1(0)$ & $1(0)$ \\
\hline Highest blood urea nitrogen & $8(0.2)$ & $6(0.2)$ \\
\hline Highest lactate level & $183(5.6)$ & $131(5.1)$ \\
\hline Highest bilirubin level & $717(21.8)$ & $561(21.8)$ \\
\hline Highest creatinine level & $8(0.2)$ & $6(0.2)$ \\
\hline Highest WBC & $12(0.4)$ & $9(0.3)$ \\
\hline Urine output & $148(4.5)$ & $120(4.7)$ \\
\hline Positive blood culture & $0(0)$ & $0(0)$ \\
\hline Documented pathogen & $0(0)$ & $0(0)$ \\
\hline Support therapies & $0(0)$ & $0(0)$ \\
\hline Renal replacement therapy & $0(0)$ & $0(0)$ \\
\hline Mechanical ventilation & $0(0)$ & $0(0)$ \\
\hline
\end{tabular}

Echo, echocardiography; SAPS, Simplified Acute Physiology Score; SOFA, Sequential Organ Failure Assessment; CNS, central nervous system; CVP, central venous pressure; MAP, mean arterial pressure; WBC, white blood cell. 


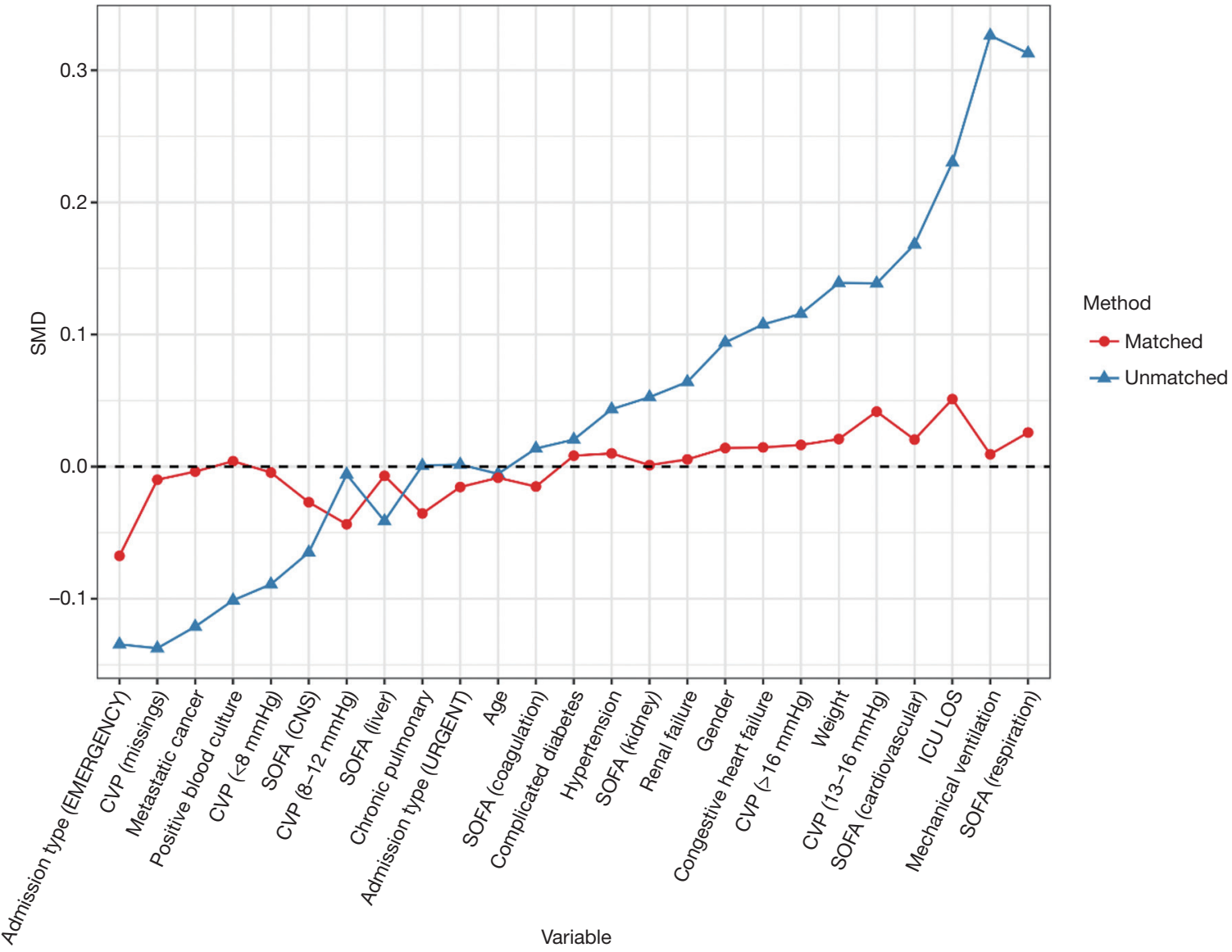

Figure S1 Standardized mean difference (SMD) of variables before and after matching. SOFA, sequential organ failure assessment; CVP, central venous pressure; CNS, central nervous system; ICU, intensive care unit; LOS, length of stay. 